\title{
Electrically Tunable Binary-Phase Fresnel Lens Based on Polymer Dispersed Liquid Crystal
}

\author{
Hui LI $^{1,2,4}$, Fan PAN ${ }^{3}$, Yuntao WU ${ }^{1,2}$, Yanduo ZHANG ${ }^{1,2}$, Xiaolin XIE $^{4}$ \\ ${ }^{1}$ School of Computer Science and Engineering, Wuhan Institute of Technology, Wuhan 40073, P. R. China \\ ${ }^{2}$ Hubei Key Laboratory of Intelligent Robot, Wuhan 40073, P. R. China \\ ${ }^{3}$ College of Post and Telecommunication, Wuhan Institute of Technology, Wuhan 40073, P. R. China \\ ${ }^{4}$ School of Chemistry and Chemical Engineering, Huazhong University of Science and Technology, Wuhan 40073, P. R. \\ China
}

crossref http://dx.doi.org/10.5755/j01.ms.23.2.16317

Received 30 September 2016; accepted 01 December 2016

\begin{abstract}
This is a proposal for a Fresnel lens with an electrically tunable binary-phase made of polymer dispersed liquid crystal (PDLC), which has relatively fast response time and low applied voltage. Simple fabrication is the major advantage of the proposed method. In this study, NOA65 and E7 were utilized with weight ratios of $60 \mathrm{wt} . \%: 40 \mathrm{wt} . \%$. There was also the utilization of a relatively low intensity UV-light, $0.53 \mathrm{~mW} / \mathrm{cm}^{2}$. The duration time of exposure was about 30 hours. The performance improvement of the Fresnel lens resulted from the infiltration of large LC droplet into the PDLC film. The phenomenon of black cross strip patterns could be explained with the use of the electro-hydrodynamics theory. The diffraction efficiency of the proposed lens was from $31.1 \%$ to $41 \%$ with the changes of externally applied voltage. This work presents an effective approach to get relatively complete phase separation in PDLC. The proposed method also provides great potential in developing high performance Fresnel lens.

Keywords: binary-phase Fresnel lens, polymer dispersed liquid crystal, phase separation, electrically tunable focal length.
\end{abstract}

\section{INTRODUCTION}

Fresnel lenses have been studied for many years and are widely applied in many fields, such as optical communication, optical information processing, image processing, and space navigation [1-8]. Both static and tunable Fresnel lenses have been reported in the past years $[9,10]$. Conventional Fresnel lenses are fabricated by some complicated technologies. The primary fabricated methods are respectively electron-beam writing and thin film deposition. However, those mainstream methods cause some disadvantages, such as non-adjustable focal lengths, expensive costs, and complicated fabrication. There is a great need for developing adjustable optical elements and a more cost efficient and simplified fabrication method. Liquid crystal (LC) is a remarkable electro-optical material, which has already been widely utilized in fabricating different variable optical devices. With the use of LC, those optical devices could be conveniently tunable by the externally applied voltage. The advantage of adopting LC material is being researched in regards to its use with Fresnel lenses [11].

A binary-phase Fresnel lens is a special one among different kinds of Fresnel lenses. Generally, it could be fabricated by an electron beam lithography method. However, the adopted method is normally expensive and complicated. Usually, LC material is utilized in order to solve those above mentioned problems. With the use of LC, there are some ordinary techniques for fabricating binary-phase Fresnel lenses. The method of electron beam

\footnotetext{
${ }^{*}$ Corresponding author. Tel.: +86-27-87992007; fax: +86-27-87992007

E-mail address: lihui00317@163.com (X. Xie)
}

lithography is utilized to form a zone plate on the indiumtin-oxide (ITO) electrode [12]. Another technique requires the making of two neighbouring zones with orthogonal LC directors [13]. Thus, those proposed methods are still complicated and expensive. This paper proposes a relatively simple method to fabricate the binary-phase Fresnel lens.

A relatively simple method is proposed in this study based on improvements of the conventional method of UV-light irradiating an optical photo-mask with zone plate patterns. In this method, both LCs and UV-monomers are adopted. The major improvement of the proposed method is to apply a relatively low intensity UV-light. The intensity of UV-light is so low that LCs and UV-monomers could have a relatively complete phase separation. Under the UV-light intensity, the relatively large LC droplets are allowed to infiltrate the PDLC film. The features of the binary-phase Fresnel lens would be quite different due to the fact that the LC droplets are larger than the ones made by the conventional method. As is well known, the bigger LC droplets would have faster response time and lower applied voltage than the smaller LC droplets at the same conditions. The refractive index of LC can be changed through the use of an externally applied voltage. An externally applied voltage would also provide variation in the diffraction efficiency and focusing features of the proposed lens. Another advantage of the proposed method is the singular irradiation time, which is less than the conventional method. The present work leads to an effectively approach to get relatively complete phase separation in PDLC and also represents the advantages of adopting the proposed method. 
In this paper, the second section presents the fabrication workflow of the proposed Fresnel lens. The third section shows some experimental results, which are used to prove the features of the Fresnel lens and discuss some of its classic, electro-optical features. The last section is the conclusion of the proposed Fresnel lens.

\section{FABRICATION}

Respectively, material preparation, empty cell fabrication, material filling, and UV-light irradiation are the basic fabrication steps of the binary-phase Fresnel lens with polymer dispersed liquid crystal (PDLC). When fabricating the Fresnel lens, the most important step is to acquire PDLC. In this study, the weight ratios between LCs (from Merck Co., E7, $n_{\mathrm{o}}=1.5216, n_{\mathrm{e}}=1.7462$, $\Delta n=0.2246$ ) and UV-monomers (NOA 65, $n_{\mathrm{p}}=1.524 @ 25{ }^{\circ} \mathrm{C}$ ) are 60 wt.\%: 40 wt.\%. Different weight percentages were measured with a precision electronic scale and then poured into a brown bottle and processed in a dark room. Originally, the two materials were mixed by an ultrasonic shaker. However, in order to acquire a uniform mixture, the gyrotron shaker was subsequently utilized. In order to fabricate the empty cell, two indium-tin-oxide (ITO) glass substrates sandwiched with $14 \mu \mathrm{m}$ spacers were utilized. Through gravity and capillary effects, the mixture was poured into an empty cell. The gaps of the empty cell were sealed by AB glues. Then, the cell was placed in UV light with an optical photo-mask of Fresnel zone plate. The more specific details about the optical photo-mask are given in the later sections. The intensity of UV light was chosen as $0.53 \mathrm{~mW} / \mathrm{cm}^{2}$, which was a relatively low light intensity compared to the conventional method. In order to achieve completion of the phase separation between LCs and UVmonomers, the speed under this condition is required to be slow. Thereafter, the relatively large LC droplets could be obtained, as proven by later experiments. The duration of exposure was about 30 hours with a singular irradiation time, which is less than one in the normal method. After that, the sample could be acquired. The schematic figure of fabricating method is shown in Fig. 1. It shows that the presented method is relatively simple.

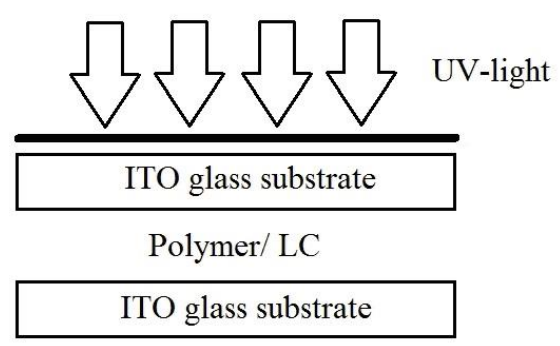

Fig. 1. The method for fabricating the Fresnel lens with an optical photo-mask

\section{EXPERIMENTAL RESULTS AND DISCUSSION}

The experiments in this section will prove the cost effective and efficient qualities of the proposed Fresnel lens. The design principle is firstly presented, then followed by the major features.

Fig. 2 demonstrates an experimental setup for measuring the classic, optical features of the proposed Fresnel lens. The sample was set on an optical displacement platform. He-Ne laser, 633nm, was utilized to illuminate this sample. The transmitted light was collected by a CCD (3 mega pixel). The detected data was analyzed by a computer.

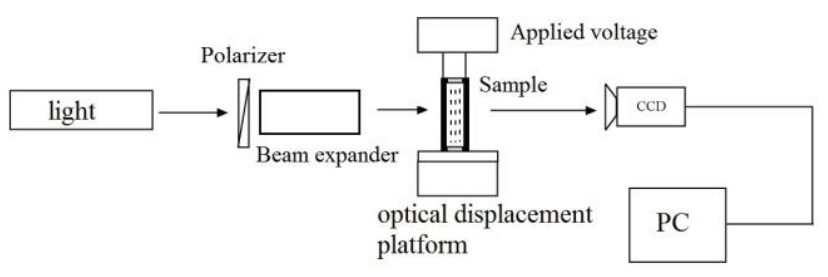

Fig. 2. Experimental setup

At first, the optical photo-mask of a Fresnel zone plate is the key point of the design of binary-phase Fresnel lenses. The Fresnel zone plate is an optical device used to focus light exhibiting wave character. However, unlike glass lenses, the Fresnel zone plate uses diffraction instead of refraction or reflection. Based on analysis by Fresnel, the Fresnel zone plate's focusing ability is an extension of the Arago spot phenomenon. This phenomenon was caused by diffraction from an opaque disc. The Fresnel zone plate consists of a set of radially symmetric rings, known as Fresnel zones, which alternate between opaque and transparent. Light transmitting through the Fresnel zone plate will diffract around the opaque zones. The zones can be spaced and the light constructively diffracted, so that it interferes at the desired focus and creates an image. The radius of the Fresnel zone plate is about

$$
R_{n}=\sqrt{n} R_{1}
$$

where $n$ is the zone number, $R_{\mathrm{n}}$ is the radius of the $n^{\text {th }}$ zone, and $R_{1}$ is the radius of the innermost radius. $R_{1}$ is chosen as $2 \mathrm{~mm}$ in this work. The even, concentric, circular region, which is originally covered, is designed to be a penetration structure of a phase difference with an adjacent, odd, concentric circle. The concentric circles are all transparent when the phase difference is $\pi$. The above mentioned structure parameters could be the design of the optical photo-mask of the Fresnel zone plate [11]. Second, the internal morphology of the Fresnel lens is a vital component to the advantages of the proposed lens. The principle of structural formation in binary-phase Fresnel lenses lies in phase separation of PDLC. This experiment will discuss the phase separation between LCs and UVmonomers and its influencing factors. As above mentioned, the PDLC was made up of E7 $60 \mathrm{wt} \%$ and NOA65 $40 \mathrm{wt} . \%$. The mixture was processed respectively by an ultrasonic shaker and a gyrotron shaker. The intensity of UV light was $0.53 \mathrm{~mW} / \mathrm{cm}^{2}$ and the exposure time was about 30 hours. The molecular weight of prepolymers increases through the poly-condensation reaction and light initiated polymerization. When the size reaches the threshold, the mutual solubility reduces and the phase separation occurs. At the same time, LC droplets are formed and gradually grow up. Finally, PDLC film is 
formed. Fig. 3 a shows the polarizing image of the PDLC film at $5 \mathrm{Vrms}$, which has a minimum scale of $100 \mu \mathrm{m}$. The dark areas demonstrate polymer-rich regions where the monomers were undergoing polymerization. Furthermore, the light areas demonstrated LC-rich and LCs molecules. This experiment determined that there were obviously black cross strip patterns ('+') inside the LC droplets, as shown in Fig. 3 a. This is a very interesting phenomenon. The reason for its formation can be explained by an electro-hydrodynamics effect [14]. The features of LC molecules such as electrically, viscosity, elastic, temperature, and electric field frequency all have a relationship with the externally applied voltage. Correspondingly, the phenomenon of ion flow also occurs under the externally applied voltage. At this time, the electrical conductivity of LC molecules has a great relationship with the ion flow. Due to the action of electrolysis, positive and negative ions in the cell produce an uneven spatial distribution of solubility gradient. Every vortex generated under the separation of electric field is perpendicular to the central axis of the direction of the electrode, as shown in Fig. 3 b. The cell is a closed space without any compression. The results of the PDLC can be clearly seen from the polarizing microscope. In micro view, as the electro-hydrodynamics effect happens inside the PDLC film, the alignment of LC molecules is distorted, resulting in different optical properties. The above mentioned theory could be proven by measuring the polarized image.
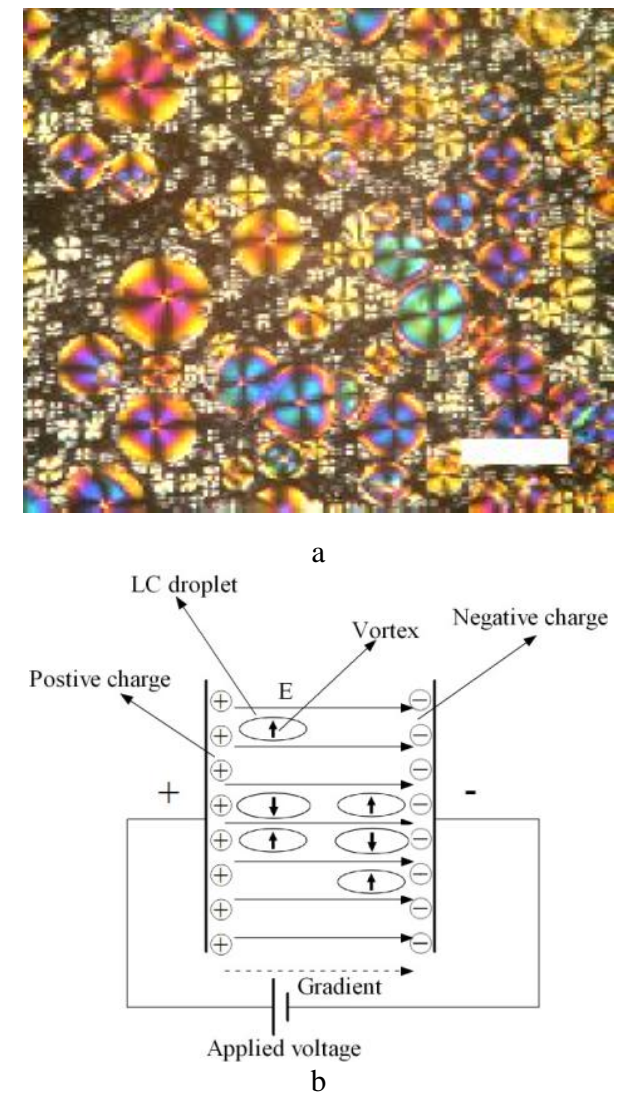

Fig. 3. Black cross strip patterns ('+') inside the LC droplets: a-polarizing microscope image of the PDLC film under the external applied voltage of $5 \mathrm{Vrms}$, and the scale is about $100 \mu \mathrm{m} ; \mathrm{b}$-schematic image of electrohydrodynamics effect
In conclusion, the electro-hydrodynamics effect is a very useful theory to explain LC morphology in the PDLC under the externally applied voltage. Then, some classic, electro-optical features of the proposed Fresnel lens are presented. The difference of the proposed lens as compared to conventional methods exists in the relatively low intensity UV light that is utilized. Generally, the UV-light is relatively strong so that the speed of the formation is relatively fast. However, that leads to a small size of LC droplets. Fig. 4 a shows the internal, polarizing image of the proposed Fresnel lens without externally applied voltage.

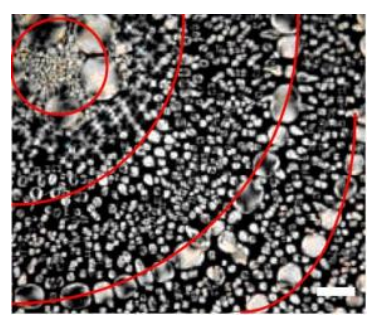

a

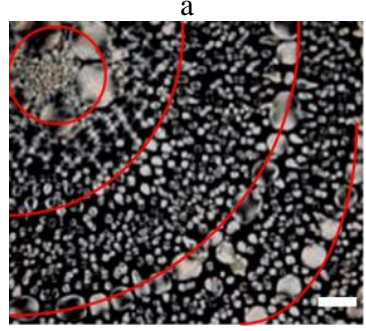

b
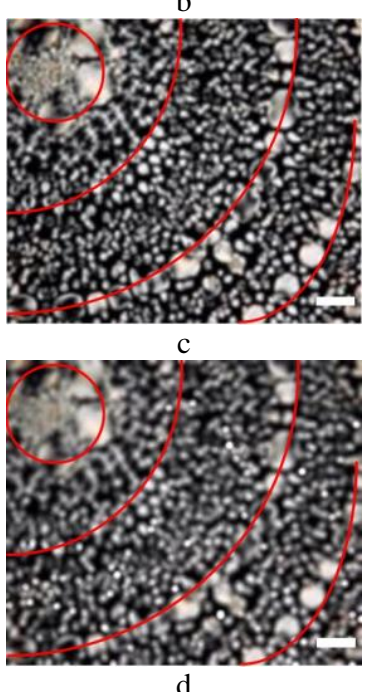

d

Fig. 4. Polarizing microscope image of the proposed Fresnel lens with the PDLC film, where a red line means the radially symmetric rings of the zone plate, and the scale is about $100 \mu \mathrm{m}: \mathrm{a}-$ is without applied voltage; $\mathrm{b}-$ is at $8.5 \mathrm{Vrms}$; $\mathrm{c}-$ is at $15 \mathrm{Vrms} ; \mathrm{d}-$ is at $25 \mathrm{Vrms}$

The image makes it clear that the size of the large LC droplet are about $100 \mu \mathrm{m}$, which is significantly larger than droplets produced by other methods. When the externally applied voltage turns on, the electro-hydrodynamics effect occurs in the PDLC. At 8.5 Vrms, black cross strip patterns appear in the PDLC, as shown in Fig. 4 b. The black cross strip patterns are due to the LC molecules that are affected by the externally applied voltage. The director of LC molecules is pulled to a parallel electric field direction and perpendicular to ITO glass substrates. While the applied 
voltage increases, the measured images become slowly blurred, as shown in Fig. $4 \mathrm{c}$ and d. This phenomenon can be explained by the above mentioned theory. A major factor contributing to this formation is the vortex caused by the electric field.

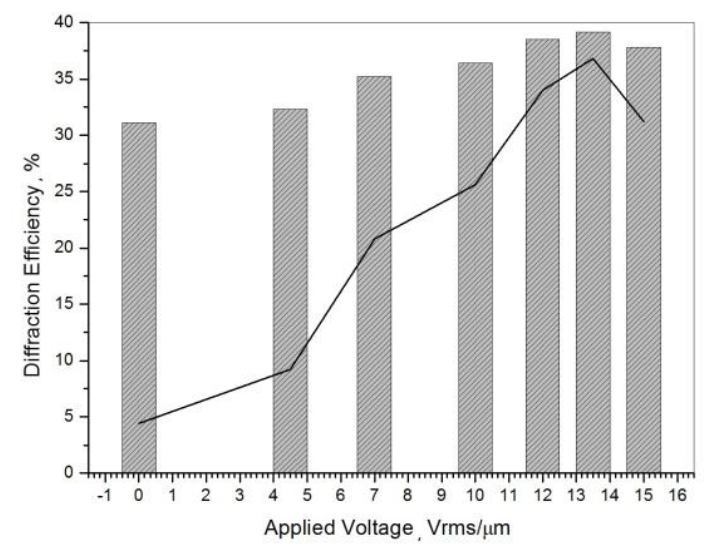

Fig. 5. Diffraction efficiency of the proposed Fresnel lens under different applied voltages

The experimental set up, as shown in Fig. 2, is utilized in order to measure the other optical features of the proposed Fresnel lens. The sample is set at $6 \mathrm{~mm}$ away from the CCD. For a binary-phase Fresnel lens, the primary focal length is $f=r^{2} / \lambda$, where $f$ is the primary focal length, $r$ is the innermost radius and $\lambda$ is the incident light wavelength. The primary focal length was computed to be $6.32 \mathrm{~mm}$ for the incident $\mathrm{He}-\mathrm{Ne}$ laser. Based on the theory of Fresnel lens, it has many focal lengths, such as $f$, $f / 3, f / 5 \ldots[15,16]$. However, because of the focusing effect, the primary focal length has the greatest energy. The diffraction efficiency of those focal lengths is $E_{\mathrm{n}}=\operatorname{sinc}^{2}(n / 2)$, where $n= \pm 1, \pm 3, \pm 5, \ldots$, and $E_{\mathrm{n}}$ is the diffraction efficiency of the Fresnel lens $[15,16]$. Thus, the theoretical value of the diffraction efficiency is about $41 \%$. However, the measured value of the diffraction efficiency is below $41 \%$, as shown in Fig. 5. At $0 \mathrm{~V}$, there has already been a Fresnel lens because phase separation is completed even without externally applied voltage. That is due to the fact that the even zones are opaque, which causes a loss of parts of the incident light energy. As it is known, the Fresnel lens is made up of LCs and polymers, while the majority are LCs. Thus, the Fresnel lens is an electrically tunable device. With the use of the experimental setup in Fig. 2, the focal length of the proposed Fresnel lens could be evaluated. The two dimensional image of the focusing state under different externally applied voltages could be acquired with a beam profiling camera from Dataray Inc., WinCamD UCD12. From the measured images in Fig. 6, the focusing states are clearly presented. At different external applied voltages, the focusing states are slightly different. That proves that the proposed Fresnel lens could be tunable by the externally applied voltage.

Finally, if the PDLC film have the same components, the response time of the proposed Fresnel lens under different intensities UV-light can be estimated, as shown in Fig. 7. The recorded instrument, EOT-01, produced by Changchun Institute of Optics, Fine Mechanics and Physics, Chinese Academy of Sciences, is used to measure the response time of the Fresnel lens. The contrast is in the results presented under the condition of different intensities of UV-light. It is obviously shown that the low intensity UV-light can affect the response time of the proposed Fresnel lens.
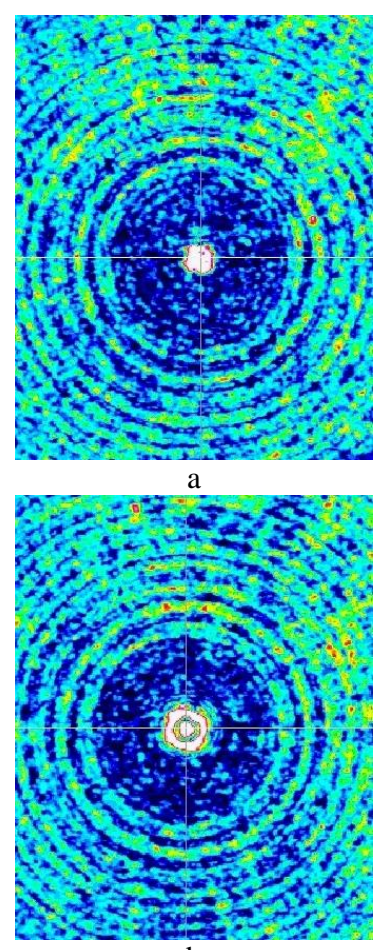

b

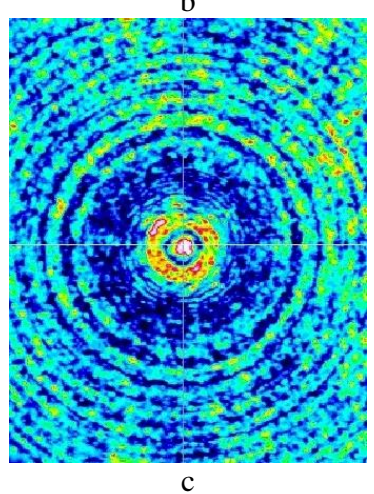

Fig. 6. The transmittance light intensity under different external applied voltages at $6 \mathrm{~mm}$ away from the CCD: a-is about $5 \mathrm{Vrms}$; $\mathrm{b}$-is about $10 \mathrm{Vrms}$; $\mathrm{c}$-is about $15 \mathrm{Vrms}$

The response time could decrease at the low intensity UV-light condition (chosen $0.53 \mathrm{~mW} / \mathrm{cm}^{2}$ for 30 hours) compared to the high intensity UV-light condition (chosen $2.5 \mathrm{~mW} / \mathrm{cm}^{2}$ for 15 hours). The range of decrease about $\tau_{\text {on }}$ is from $18.0 \mathrm{~ms}$ to $1.5 \mathrm{~ms}$. On the other hand, the range of decrease about $\tau_{\text {off }}$ is from $14.2 \mathrm{~ms}$ to $10.5 \mathrm{~ms}$. The response time $\left(\tau_{\text {on }}+\tau_{\text {off }}\right)$ is from $32.2 \mathrm{~ms}$ to $12.0 \mathrm{~ms}$. The specific reason is that the larger LC droplets can be acquired under the relatively low intensity condition. Therefore, the response time of the LC device obviously improved when the other conditions were the same. In conclusion, larger LC droplets could get under the condition of a low intensity UV-light. The Fresnel lens under the condition of a low intensity UV-light has a relatively fast response time compared to a high intensity UV-light condition. 


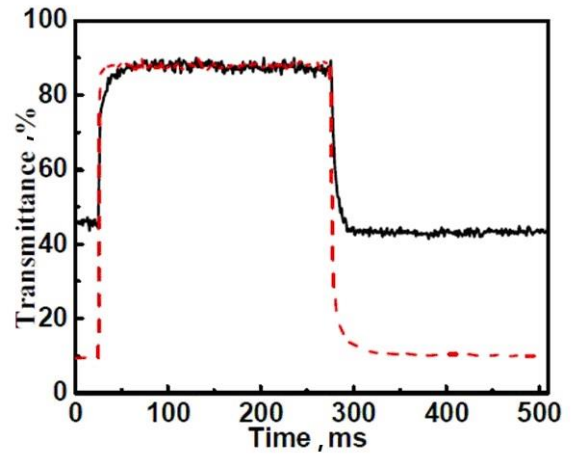

Fig. 7. The response time of the proposed Fresnel lens under different intensities of UV-light, where the red line means the relatively low intensity UV-light condition (chosen $0.53 \mathrm{~mW} / \mathrm{cm}^{2}$ for 30 hours) and the black line means the relatively high intensity UV-light condition (chosen $2.5 \mathrm{~mW} / \mathrm{cm}^{2}$ for 15 hours)

\section{CONCLUSIONS}

This paper proposes a new type Fresnel lens with a PDLC film, which is fabricated by a relatively low intensity UV-light. When the PDLC is utilized in fabricating the lens, the result is a new kind of Fresnel lens with a simple, efficient fabrication workflow. The simplicity and cost efficiency of the proposed method sets it apart from other conventional methods. The relatively low intensity UV-light is very helpful for improving its response time and applied voltage. The special phenomenon formed in the PDLC, black cross strip patterns, could be very well explained based on the electrohydrodynamics effect. The major factor of forming larger LC droplets is discussed and analyzed. And related experiments about the electro-optical features of the proposed Fresnel lens based on PDLC are presented in this work. The proposed method has great potential in developing high-performance Fresnel lenses. However, the binary-phase Fresnel lens has relatively low diffraction efficiency only above $31.1 \%$ and below $41 \%$, which leaves room for further improvement.

\section{Acknowledgments}

This work was supported by National Natural Science Foundation of China (61671337), a grant from the Research Foundation of Education Bureau of Hubei Province China (B2016489), a grant from the Natural Science Foundation of Hubei Province of China (2014CFB771), China Postdoctoral Science Foundation (2014M562017), the Science Foundation of the Wuhan Institute of Technology (Q201601), and the Open Foundation for Hubei Key Laboratory of Intelligent Robot (HBIR 201605).

\section{REFERENCES}

1. Canning, J., Sommer, K., Huntington, S., Carter, A. Silica-Based Fiber Fresnel Lens Optics Communications 199 (5-6) 2001: pp. 375-381.

2. Dammann, H. Blazed Synthetic Phase-Only Holograms Optik 31 (1) 1970: pp. 95-104.
3. Jahns, J., Walker, S.J. Two-Dimensional Array of Diffractive Microlenses Fabricated by Thin-Film Deposition Applied Opics 29 (7) 1990: pp. 931-936.

4. Wang, X.Q., Fan, F., Du, T., Tam, A.M.W., Ma, Y., Srivastava, A.K., Chigrinov, V.G., Kwok, H.S. Liquid Crystal Fresnel Zone Lens Based on Single-Side-Patterned Photoalignment Layer Applied Optics 53 (10) 2014: pp. 2026-2029. https://doi.org/10.1364/AO.53.002026

5. Bencheikh, A., Fromager, M., Ameur, K. Generation of Laguerre-Gaussian LGp0 Beams Using Binary Phase Diffractive Optical Elements Applied Optics 53 (21) 2014: pp. $4761-4767$. https://doi.org/10.1364/AO.53.004761

6. Siiman, L., Lumeau, J., Glebov, L. Phase Fresnel Lens Recorded in Photo-Thermo-Refractive Glass by Selective Exposoure to Infrared Ultrashort Laser Pulses Optics Letters 34 (1) 2009: pp. 40-42.

7. Rong, N., Li, Y., Li, X., Zhou, P., Liu, S., Lu, J., Su, Y. Polymer-Stabilized Blue-Phase Liquid Crystal Fresnel Lens Cured With Patterned Light Using a Spatial Light Modulator Journal of Display Technology 12 (10) 2016: pp. $1008-1012$.

8. Lin, S.H., Huang, B.Y., Li, C.Y., Yu, K.Y., Chen, J.L., Kuo, C.T. Electrically and Optically Tunable Fresnel Lens in a Liquid Crystal Cell With a Rewritable Photo Conductive Layer Optical Material Express 6 (7) 2016: pp. 2229-2235.

9. Ferstl, M., Frisch, A. Static and Dynamic Fresnel Zone Lenses for Optical Interconnections Journal of Modern Optics 43 (7) 1996: pp. 1451-1462.

10. Mingtao, L., Wang, J., Zhuang, L., Chou, S.Y. Fabrication of Circular Optical Structures with a $20 \mathrm{~nm}$ Minimum Feature Size Using Nanoimprint Lithography Applied Physics Letters 76 (6) 2000: pp. 673-675. https://doi.org/10.1063/1.125896

11. Ren, H., Fan, Y.H., Wu, S.T. Tunable Fresnel Lens Using Nanoscale Polymer-Dispersed Liquid Crystals Applied Physics Letters 83 (8) 2003: pp. 1515-1517. https://doi.org/10.1063/1.1604943

12. Chou, S.Y., Zhuang, L. Lithographically Induced SelfAssembly of Periodic Polymer Micropillar Arrays Journal of Vacuum Science and Technology B 17 (6) 1999: pp. 3197-3202.

13. Kim, D.W., Yu, C.J., Kim, H.R., Kim, S.J., Lee, S.D. Polarization-Insensitive Liquid Crystal Fresnel Lens of Dynamic Focusing in an Orthogonal Binary Applied Physics Letters 88 (20) 2006: pp. 203505-1-203505-3.

14. Dickey, M.D., Collister, E., Raines, A., Tsiartas, P., Holcombe, T., Sreenivasan, S.V., $\quad$ Bonnecaze, R.T., Willson, C.G. Photocurable Pillar Arrays Formed via Electrohydrodynamic Instabilities Chemistry of Materials 18 (8) 2006: pp. 2043-2049. https://doi.org/10.1021/cm052592w

15. Williams, G., Powell, N.J., Purvis, A., Clark, M.G. Electrically Controllable Liquid Crystal Fresnel Lens Proceeding of SPIE 1168 1989: pp. 352-357. https://doi.org/10.1117/12.962998

16. Patel, J.S., Rastani, K. Electrically Controlled PolarizationIndependent Liquid-Crystal Fresnel Lens Arrays Optics Letters $16(7)$ 1991: pp. 532-534. https://doi.org/10.1364/OL.16.000532 